\title{
INFINITELY DISTRIBUTIVE ELEMENTS IN POSETS
}

\author{
Vera Lazarević* and Andreja Tepavčević
}

\begin{abstract}
Infinitely distributive and codistributive elements in posets are studied. It is proved that an element $a$ in a poset $P$ has these properties if and only if the image of $a$ has the corresponding properties in the Dedekind MacNeille completion of $P$. An application of the order theoretical results to a poset of weak congruences is presented.
\end{abstract}

\section{Preliminaries}

\subsection{Special elements in lattices}

An element $a$ of a lattice $L$ is infinitely distributive iff for every family $\left\{x_{i} \mid i \in I\right\} \subseteq L$ :

$$
a \vee\left(\bigwedge_{i \in I} x_{i}\right)=\bigwedge_{i \in I}\left(a \vee x_{i}\right)
$$

An element $a$ which satisfies the dual law is called infinitely codistributive.

Elements satisfying the corresponding laws with finite families $I$ usually are called distributive (or codistributive).

In paper [20] the relationship between infinite and finite distributive (codistributive) elements has been investigated.

Element $a \in L$ is infinitely codistributive if and only if the mapping $m_{a}: L \longrightarrow a \downarrow$ defined by $m_{a}(x)=a \wedge x$ is a complete lattice homomorphism (homomorphism which is compatible with all suprema and infima). This homomorphism induces a complete congruence on $\mathrm{L}$ [18]. Moreover, if lattice $L$ is algebraic, classes of the induced congruence always have the top elements.

AMS (MOS) Subject Classification 1991. Primary: 04A72, 06A12.

Key words and phrases: Infinite distributive, elements, infinite codistributive elements, congruence, $\omega$-stable, complete congruence.

*Research supported by Science Fund of Serbia under Grant 1457. 
The dual complete homomorphism connected with the completely distributive elements will be denoted by $n_{a}$.

For a poset $(P, \leq)$ and $X \subseteq P$ we introduce the following notions and notations.

Let $L_{P}(X)$ be the set of all lower bounds of $X$ in $P$, and $U_{P}(X)$ the set of all upper bounds:

$$
\begin{aligned}
& L_{P}(X)=\{y \in P \mid y \leq x \text { for all } \quad x \in X\}, \\
& U_{P}(X)=\{y \in P \mid x \leq y \text { for all } \quad x \in X\} .
\end{aligned}
$$

In no confusion can occur, subscripts will be omitted and we shall write $\mathrm{L}(\mathrm{X})$ and $\mathrm{U}(\mathrm{X})$.

If $X=\left\{x_{1}, \ldots x_{n}\right\}$ is finite then instead of $L(X)$ and $U(X)$, we also use the notation $L\left(x_{1}, \ldots, x_{n}\right)$ and $U\left(x_{1}, \ldots, x_{n}\right) . L(X \cup Y)$ will be denoted by $L(X, Y)$, and similarly $U(X \cup Y)$, by $U(X, Y)$.

Throughout the paper $D M(P)$ denotes the Dedekind-McNeille completion of $P$. In this context, $G(P)$ is the sublattice of $D M(P)$ generated by the set $\{L(x) \mid x \in P\}$. This lattice is called, according to [6], characteristic lattice of poset $P$.

Let $e_{P}$ be a natural mapping from a poset $P$ to its Dedekind-McNeille completion of $P$.

$$
e_{P}: P \rightarrow D M(P) \quad \text { defined by } \quad e_{P}(x):=L(x) .
$$

A mapping $f: P \rightarrow Q$ is $\omega$-stable [6] if there is a lattice homomorphism $f^{*}: G(P) \rightarrow G(Q)$ such that $e_{Q} \circ f=f^{*} \circ e_{P}$.

An equivalence on $P$ is defined to be a congruence on $P$ if it is a kernel of a $\omega$-stable mapping on $P[6]$.

Theorem 1. [6] A relation on a poset $P$ is a congruence on $P$ if and only if it corresponds to a restriction of a congruence on lattice $G(P)$.

We call a relation on a poset $P$ complete congruence if it is a restriction of a complete congruence relation on the lattice $D M(P)$ (complete congruences are equivalence relations compatible with arbitrary suprema and infima).

Throughout the paper, infima and suprema in $P$ are denoted by $i n f_{P}$ and $\sup _{P}$, and infima and suprema in lattices $D M(P)$ and $G(P)$ are denoted by $\wedge$ and $\vee$. The ordering relation in the poset and the related Dedekind MacNeille completion is denoted by the same symbol $\leq$.

The following lemma is a consequence of the fact that mapping $e_{P}$ is an order embedding. 
Lemma 1. Let $P$ be a poset and $D M(P)$ its Dedekind MacNeille completion. Then for $a \in P, X \subseteq P$,

$$
a \in U_{P}(X) \text { if and only if } e_{P}(a) \geq \bigvee_{x \in X} e_{P}(x),
$$

where $\bigvee$ is the supremum in the $D M(P)$.

The dual lemma is also valid.

\subsection{Identities in posets}

Identities on posets are introduced and studied in several papers (see e.g. [8], [12], [13]).

A partially ordered set $P$ is distributive [8] if for all $x, y, z \in P$,

$$
L(x, U(y, z))=L(U(L(x, y), L(x, z))) .
$$

It is proved by Lamerová and Rachunek ( [8]) that this condition is equivalent with its dual:

$$
U(x, L(y, z))=U(L(U(x, y), U(x, z))) .
$$

It turned out that the distributivity of a poset is connected with the distributivity of the corresponding characteristic lattice.

Theorem 2. (Niederle [12]): Poset $P$ is distributive if and only if it is a doubly dense subset of a distributive lattice.

Theorem 3. (Niederle [13]): Poset $P$ is distributive if and only if the lattice $G(P)$ is distributive.

\section{Special elements in posets}

A large class of special elements in posets has been introduced and studied in [23] and [24].

In this section we introduce notions of infinitely distributive and codistributive elements in posets. We characterize these elements by infinite (co) distributivity in the lattice $D M(P)$.

Element $a$ in a poset $P$ is infinitely distributive if and only if for every family $\left\{x_{i} \mid i \in I\right\}$ of elements from $P, U\left(a, L\left(\left\{x_{i} \mid i \in I\right\}\right)\right)=$ $U\left(L\left(\bigcup_{i \in I} U\left(a, x_{i}\right)\right)\right)$.

Element $a$ in a poset $P$ is infinitely codistributive if for every family $\left\{x_{i} \mid i \in I\right\}$ of elements from $P, L\left(a, U\left(\left\{x_{i} \mid i \in I\right\}\right)\right)=L\left(U\left(\bigcup_{i \in I} L\left(a, x_{i}\right)\right)\right)$.

Theorem 4. Element $a \in P$ is infinitely distributive in $P$ if and only if $e_{P}(a)$ is an infinitely distributive element in the lattice $D M(P)$. 
Proof. Let $a \in P$ and let $e_{P}(a)$ be an infinitely distributive element in $D M(P)$. Recall that $P$ is a double dense subset of $D M(P)$. Let

$$
t \in U\left(a, L\left(\left\{x_{i} \mid i \in I\right\}\right)\right) .
$$

Then, $t \geq a$ and for any $x \in L\left\{x_{i} \mid i \in I\right\}, t \geq x$. Therefore, $e_{P}(t) \geq e_{P}(a)$ and $e_{P}(t) \geq e_{P}(x)$ for all $x \in L\left\{x_{i} \mid i \in I\right\}$. In lattice $D M(P), e_{P}(x) \leq e_{P}\left(x_{i}\right)$, for all $i \in I$, and therefore $e_{P}(x) \leq \bigwedge_{i \in I} e_{P}\left(x_{i}\right)$. Hence, $e_{P}(t) \geq e_{P}(x)$ for all $x \leq \bigwedge_{i \in I} x_{i}$. Since $\bigwedge_{i \in I} e\left(x_{i}\right)=\bigvee\left\{e(x) \mid e(x) \leq \bigwedge_{i \in I} e\left(x_{i}\right)\right\}$, we have that $e_{P}(t) \geq \bigwedge_{i \in I} e\left(x_{i}\right)$. Further on, $e_{P}(t) \geq e_{P}(a) \vee \bigwedge_{i \in I} e_{P}\left(x_{i}\right)=\bigwedge_{i \in I}\left(e_{P}(a) \vee\right.$ $\left.e_{P}\left(x_{i}\right)\right)$ by the infinite distributivity of $e_{P}(a)$ in $D M(P)$.

Let $p \in L\left(\bigcup_{i \in I} U\left(a, x_{i}\right)\right)$. Then, $p \leq q$ for every $q \in \bigcup_{i \in I} U\left(a, x_{i}\right)$, and by the similar arguments as above, $e_{P}(p) \leq e_{P}(a) \vee e_{p}\left(x_{i}\right)$. Therefore, $e_{P}(p) \leq \bigwedge_{i \in I}\left(e_{P}(a) \vee e_{P}\left(x_{i}\right)\right)$. Hence, $e_{P}(p) \leq e_{P}(t)$, and $p \leq t$. Hence, $t \in U\left(L\left(\bigcup_{i \in I} U\left(a, x_{i}\right)\right)\right)$.

Therefore, we proved $U\left(a, L\left\{x_{i} \mid i \in I\right\}\right) \subseteq U\left(L\left(\bigcup_{i \in I} U\left(a, x_{i}\right)\right)\right)$. The other inclusion is always fulfilled.

Now, we suppose that $a$ is an infinitely distributive element of $P . P$ is a double dense subset in $D M(P)$.

Firstly, we prove that $e_{P}(a) \vee \bigwedge_{i \in I} e_{P}\left(x_{i}\right)=\bigwedge_{i \in I}\left(e_{P}(a) \vee e_{P}\left(x_{i}\right)\right)$ is satisfied for all $x_{i} \in P$.

Let $y \in P$. Then, $e_{P}(y) \geq \bigwedge_{i \in I}\left(e_{P}(a) \vee e_{P}\left(x_{i}\right)\right)$ if and only if $e_{P}(y) \in U\left(\bigwedge_{i \in I}\left(e_{P}(a) \vee e_{P}\left(x_{i}\right)\right)\right)$ if and only if $y \in U\left(L\left(\bigcup_{i \in I} U\left(a, x_{i}\right)\right)\right)$ if and only if $y \in U\left(a, L\left\{x_{i} \mid i \in I\right\}\right)$ if and only if $e_{P}(y) \in U\left(e_{P}(a), \bigwedge_{i \in I} e_{P}\left(x_{i}\right)\right)$ if and only if $e_{P}(y) \geq e_{P}(a)$ and $e_{P}(y) \geq \wedge_{i \in I} e_{P}\left(x_{i}\right)$ if and only if $e_{P}(y) \geq e_{P}(a) \vee \bigwedge_{i \in I} e_{P}\left(x_{i}\right)$.

If $\left\{x_{i} \mid i \in I\right\}$ is a family of elements from $D M(P)$, then every $x_{i}$ is an infimum of a family of images of elements from $P, x_{i}=\wedge_{j \in J_{i}} e_{P}\left(z_{j}\right)$, for $z_{j} \in P$.

Therefore,

$$
\begin{aligned}
e_{P}(a) \vee \bigwedge_{i \in I} x_{i} & =e_{P}(a) \vee \bigwedge_{i \in I} \bigwedge_{j \in J_{i}} e_{P}\left(z_{j}\right)=\bigwedge_{i \in I} \bigwedge_{j \in I_{j}}\left(e_{P}(a) \vee e_{P}\left(z_{j}\right)\right) \geq \\
\geq \bigwedge_{i \in I}\left(e_{P}(a) \vee \bigwedge_{j \in I_{j}} e_{P}\left(z_{j}\right)\right) & =\bigwedge_{i \in I}\left(e_{P}(a) \vee x_{j}\right) .
\end{aligned}
$$

The other inequality is always satisfied.

The dual theorem is also satisfied. 
Theorem 5. Element $a \in P$ is infinitely codistributive if and only if $e_{P}(a)$ is an infinitely codistributive element in the lattice $D M(P)$.

By the Lemma 1 in [18] we obtain following consequences:

Corollary 1. Element $a \in P$ is infinitely distributive if and only if relation $\theta_{a}$ on $P$, defined by

$$
x \theta_{a} y \text { if and only if } e_{P}(x) \vee e_{P}(a)=e_{P}(y) \vee e_{P}(a)
$$

is a complete congruence on poset $P$.

Corollary 2. Element $a \in P$ is infinitely codistributive if and only if relation $\theta_{a}$ on $P$, defined by

$$
x \theta_{a} y \text { if and only if } e_{P}(x) \wedge e_{P}(a)=e_{P}(y) \wedge e_{P}(a)
$$

is a complete congruence on poset $P$.

\subsection{Weak congruence lattice}

In this section we recall the notion of weak congruences which will serve as a justification of introduction of the terms of distributive and codistributive elements in posets.

Let $\mathcal{A}=(A, F)$ be an algebra. Let $C w \mathcal{A}$ be a set of all weak congruences (symmetric and transitive and compatible relations) on $\mathcal{A}$. $(C w \mathcal{A}, \subseteq)$ is an algebraic lattice. It is a lattice of all congruences on all subalgebras together with the empty set in case when there is no nullary operation in the similarity type.

The diagonal relation $\Delta$ is always an infinitely codistributive element in $C w \mathcal{A}$. The filter $\Delta \uparrow$ is the congruence lattice $\operatorname{Con} \mathcal{A}$, and the ideal $\Delta \downarrow$ is isomorphic with the subuniverse lattice $\operatorname{Sub} \mathcal{A}$.

The top elements of the congruence classes determined by the homomorphism $m_{\Delta}: x \mapsto x \wedge \Delta$ are squares of subuniverses.

An algebra $\mathcal{A}$ has the congruence intersection property (CIP) iff $\Delta$ is a distributive element in the lattice $C w \mathcal{A}$.

An algebra $\mathcal{A}$ has the infinite congruence intersection property $\left({ }^{*} \mathrm{CIP}\right)$ if and only if for an arbitrary family of weak congruences $\left\{\rho_{i} \mid i \in I\right\}$,

$$
\Delta \vee\left(\bigwedge_{i \in I} \rho_{i}\right)=\bigwedge_{i \in I}\left(\Delta \vee \rho_{i}\right)
$$




\subsection{Weak congruences under different order}

Let $C w \mathcal{A}$ be a set of all weak congruences of an algebra $\mathcal{A}$, and $\Delta$ the diagonal relation.

Let $\rho, \theta$ be two weak congruences, and $\rho \in \operatorname{Con\mathcal {C}}, \theta \in \operatorname{Con\mathcal {B}}$. We introduce a new operation on $C w \mathcal{A}$ :

$$
\rho * \theta=\left(B^{2} \wedge \rho\right) \vee\left(C^{2} \wedge \theta\right),
$$

and $\emptyset * \theta=\emptyset$.

Use of such an operation, which is also a graphical composition was proposed by M. Ploščica in [17].

In the sequel, $(C w \mathcal{A}, \wedge, \vee)$ or $(C w \mathcal{A}, \subseteq)$ is a weak congruence lattice of an algebra $\mathcal{A}$, and $\left(C w \mathcal{A}, \leq_{*}\right)$ is the poset of weak congruences, where the relation $\leq_{*}$ is defined by the operation $*$ :

$$
\rho \leq_{*} \theta \text { if and only if } \rho * \theta=\theta .
$$

Theorem 6. [9] Let $C w \mathcal{A}$ be a weak congruence lattice, and $*$ and $\leq_{*}$ be defined as above. Then:

(i) $\Delta \leq_{*} \rho$, for all $\rho \in C w \mathcal{A}$.

(ii) If $\rho, \theta \in\left[\Delta_{B}, B^{2}\right]$, then $\rho * \theta=\rho \vee \theta$, for $B \in \operatorname{Sub} \mathcal{A}$.

(iii) $\rho \subseteq \theta$ if and only if $\rho \leq_{*} \theta$, for $\rho, \theta \in\left[\Delta_{B}, B^{2}\right]$.

(iv) The interval $\left[\Delta_{B}, B^{2}\right]_{*}$ is a lattice $\operatorname{Con} \mathcal{B}$, for $\mathcal{B} \in \operatorname{Sub} \mathcal{A}$.

(v) $B^{2} * C^{2}=B^{2} \wedge C^{2}$.

(vi) $B^{2} \leq_{*} C^{2}$ if and only if $C^{2} \subseteq B^{2}$.

(vii) $A^{2} \leq_{*} \rho$ if and only if $\rho=B^{2}$, for $\rho \in C o n \mathcal{B}$.

(viii) $C w \mathcal{A}$ is equal to the union of intervals $\left[\Delta_{B}, B^{2}\right]_{*}$, for all $B \in \operatorname{Sub} \mathcal{A}$.

(ix) The filter $A^{2} \uparrow_{*}$ is anti-isomorphic with $\operatorname{Sub} \mathcal{A}$.

(x) If $\rho \in C$ on $\mathcal{B}$, then $\rho * A^{2}=B^{2}$.

Example 1. A lattice of weak congruences $(C w \mathcal{G}, \subseteq)$ for a four-element Klein's group $\mathcal{G}$ is given in Figure 2 a). A poset of weak congruences $\left(C w \mathcal{G}, \leq_{*}\right)$ for the same group is given in Fig. $2 \mathrm{~b}$ ). 


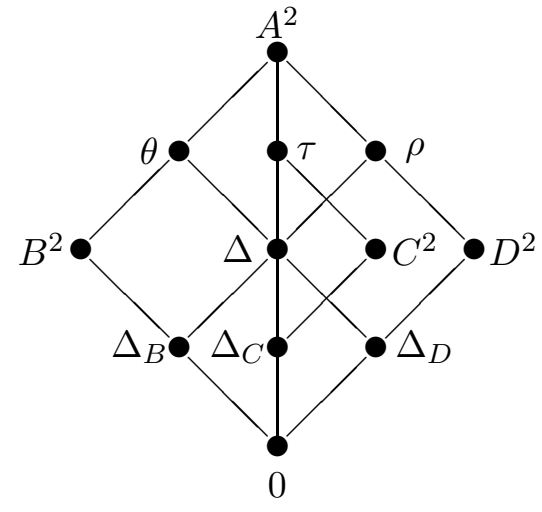

$(C w \mathcal{G}, \subseteq)$

Figure 1 a)

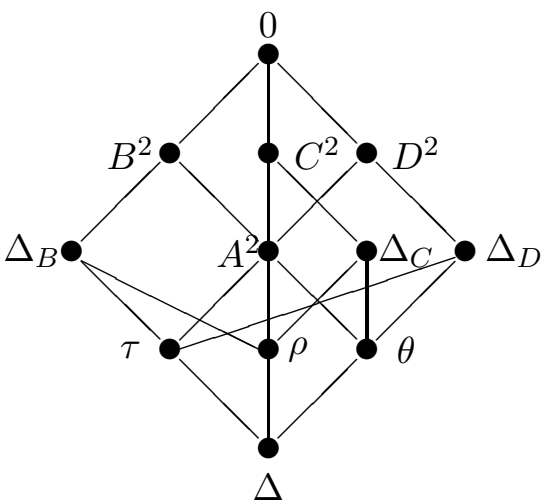

$\left(C w \mathcal{G}, \leq_{*}\right)$

Figure $1 \mathrm{~b}$ )

\section{Special elements in poset of weak congruences}

Let $A$ be an algebra, and $\left(C w \mathcal{A}, \leq_{*}\right)$ the poset of weak congruences.

Being the bottom element, the diagonal relation $\Delta$ is always an infinitely distributive and infinitely codistributive element in this poset.

Lemma 2. In the poset $\left(C w \mathcal{A}, \leq_{*}\right)$, for every $\rho \in \operatorname{Con\mathcal {B},} B \in \operatorname{Sub} \mathcal{A}$

$$
\sup \left\{A^{2}, \rho\right\}=B^{2} .
$$

Proof. Since $A^{2} * \rho=B^{2}, B^{2}$ is an upper bound for elements $A^{2}$ and $\rho$. Let $\theta \in C w \mathcal{A}$ be another upper bound, i.e., let $A^{2} \leq_{*} \theta$ and $\rho \leq_{*} \theta$. Let $\theta \in \operatorname{Con\mathcal {C}}$, for $C \in \operatorname{Sub} \mathcal{A}$. Hence,

$$
\theta=A^{2} * \theta=\left(A^{2} \wedge \theta\right) \vee\left(A^{2} \wedge C^{2}\right)=\theta \vee C^{2}=C^{2},
$$

and

$$
C^{2}=\rho * C^{2}=\left(B^{2} \wedge C^{2}\right) \vee\left(\rho \wedge C^{2}\right)=B^{2} \wedge C^{2} .
$$

Thus, $C^{2} \subseteq B^{2}$ and $C \leq B$. By the Theorem 6 . (vi), $B^{2} \leq_{*} C^{2}$ and $B^{2}$ is the required supremum.

Lemma 3. In the poset $\left(C w \mathcal{A}, \leq_{*}\right)$, for every family $\left\{B_{i} \in \operatorname{Sub} \mathcal{A} \mid i \in I\right\}$

$$
\inf _{i \in I} B_{i}{ }^{2}=\bigvee_{i \in I} B_{i}{ }^{2}
$$

where the operation $\vee$ at the right is the supremum in the weak congruence lattice. 
Proof. By Theorem 6., $\bigvee_{i \in I} B_{i}{ }^{2}$ is a maximim lower bound for elements $\left\{B_{i} \mid i \in I\right\}$. Suppose that $\rho \in C o n \mathcal{D}$ is another lower bound for elements $B_{i}$ for $i \in I: \rho \leq_{*} B_{i}$, for all $i \in I$. Hence, $B_{i} \subseteq D$, for all $i \in I$ and thus $\bigvee_{i \in I} B_{i}{ }^{2} \subseteq D$. Therefore, $\rho *\left(\bigvee_{i \in I} B_{i}{ }^{2}\right)=\left(\rho \wedge\left(\bigvee_{i \in I} B_{i}{ }^{2}\right)\right) \vee\left(D^{2} \wedge\left(\bigvee_{i \in I} B_{i}{ }^{2}\right)\right)=$ $\left(\bigvee_{i \in I} B_{i}{ }^{2}\right)$. Hence, $\bigvee_{i \in I} B_{i}{ }^{2}$ is the required infimum.

Theorem 7. $A^{2}$ is an infinitely distributive element in poset $\left(C w \mathcal{A}, \leq_{*}\right)$.

Proof. Let $\left\{\rho_{i} \mid i \in I\right\}$ be a family of weak congruences, where $\rho_{i} \in$ $\operatorname{Con}_{i}$ for $\mathcal{B}_{i} \in \operatorname{Sub} \mathcal{A}$.

By Lemma 2., $\sup \left\{A^{2}, \rho_{i}\right\}=B_{i}^{2}$ for each $i \in I$, where suprema are considered under the ordering $\leq_{*}$.

Hence, $U\left(A^{2}, \rho_{i}\right)=B_{i}^{2} \uparrow$, for every $i \in I$. By Lemma 3

$$
U\left(L\left(\bigcup_{i \in I} U\left(A^{2}, \rho_{i}\right)\right)\right)=\left(\bigvee_{i \in I} B_{i}\right) \uparrow .
$$

On the other hand, let $\rho$ belong to $U\left(A^{2}, L\left(\left\{\rho_{i} \mid i \in I\right\}\right)\right)$. Since $\rho \geq A^{2}$, and by Theorem 6 . (vii), $\rho=D^{2}$, for some subalgebra $\mathcal{D}$. $D^{2} \geq \theta$ for any $\left.\theta \in L\left(\left\{\rho_{i} \mid i \in I\right\}\right)\right)$. We prove that $\Delta_{S} \leq \rho_{i}$ for any $i \in I$, where $S=\bigvee_{i \in I} B_{i}$. Indeed,

$$
\Delta_{S} * \rho_{i}=\left(S^{2} \wedge \rho_{i}\right) \vee\left(\Delta_{S} \wedge B_{i}^{2}\right)=\rho_{i} \vee \Delta_{B_{i}}=\rho_{i},
$$

and thus $\Delta_{S} \leq_{*} \rho_{i}$. Hence, $\left.\Delta_{S} \in L\left(\left\{\rho_{i} \mid i \in I\right\}\right)\right)$ and $D^{2} \geq_{*} \Delta_{S}$, therefore $\left(D^{2} \wedge \Delta_{S}\right) \vee\left(D^{2} \wedge S^{2}\right)=D^{2}$ and $D^{2} \leq S^{2}$, and $D \leq S$ in subalgebra lattice. By , $\rho=D^{2} \in\left(\bigvee_{i \in I} B_{i}\right) \uparrow$ and

$$
U\left(A^{2}, L\left(\left\{\rho_{i} \mid i \in I\right\}\right)\right) \subseteq U\left(L\left(\bigcup_{i \in I} U\left(A^{2}, \rho_{i}\right)\right)\right) .
$$

The other inclusion is always satisfied.

Since $A^{2}$ is an infinitely distributive element, we obtain the natural decomposition to congruence classes of the poset $\left(C w \mathcal{A}, \leq_{*}\right)$.

Corollary 3. Each block of the congruence on poset $\left(C w \mathcal{A}, \leq_{*}\right)$ induced by $\rho \mapsto \sup \left\{\rho, A^{2}\right\}$ is a congruence lattice of a subalgebra $\mathcal{B}$, where $\rho$ belongs to $C o n \mathcal{B}$. Therefore, poset $\left(C w \mathcal{A}, \leq_{*}\right)$ is the union of intervals $C o n \mathcal{B}$ which are the congruences lattices on all the subalgebras of $\mathcal{A}$.

\section{References}

[1] G. Birkhoff, Lattice theory, Amer. Math. Soc. Providence, Rhode Island, 1967.

[2] I. Chajda, B. Šešelja, A. Tepavčević, Lattices of compatible realations satisfying a set of formulas, Algebra Univers. 40 (1998) 51-58. 
[3] I. Chajda, Complemented ordered sets, Archivum Math. (Brno) 28, 25-34, 1992.

[4] B. A. Davey, H. A. Priestley, Introduction to Lattices and Order, Cambridge University Press 1990.

[5] G. Grätzer, General Lattice Theory, Akademie-Verlag Berlin, 1978.

[6] R. Halaš, Congruences on posets, Contributions to General Algebra 12, Proceedings of the Vienna Conference, June 3-6, 1999, Verlag Johannes Heyn, Klagenfurt 2000 .

[7] V.S. Kharat, B.N. Waphare, Reducibility in finite posets, Europ. J. Combinatorics (2001) 22, 197-205.

[8] J. Lamerová, J. Rachunek, Translations of distributive and modular ordered sets, Acta Univ. Palack. Olom. Math. 27, 13-23.

[9] V. Lazarević, A. Tepavčević, Weak congruences and graphical composition, Contributions to General Algebra 13, Verlag Johannes Heyn, Klagenfurt 2001, 199205.

[10] V. Lazarević, A. Tepavčević, A new ordering relation on lattices applied to weak congruences, Filomat, 15, 2001, 39-46.

[11] K. Leutöla, J. Nieminen, Posets and generalized suprema and infima, Algebra Univers. 1983, 16, No. 3, 344-354.

[12] J. Niederle, Boolean and distributive ordered sets: Characterization and Representation by Sets, Order 12, 198-210, 1995.

[13] J. Niederle, Identities in ordered sets, Order 15, 271-278, 1999.

[14] J. Niederle, On pseudocomplemented and Stone ordered sets, Order 18, 161-170, 2001.

[15] J. Niederle, On pseudocomplemented, 0-distributive and infinitely distributive ordered sets, presented at the Summer School on General Algebra and Ordered Sets, Tale.

[16] M.M. Pawar, B.N. Waphare, On Stone posets and strongly pseudocomplemented posets, Journal of Indian. Math. Soc. Vol. 68, Nos. 1-4 (2001), 91-95.

[17] M. Ploščica, Graphical compositions and weak congruences, Publ. Inst. Math. Beograd 56 (70) 1974, 34-40.

[18] B. Šešelja, A. Tepavčević, Infinitely distributive elements in the lattice of weak congruences, General Algebra 1988, Elsevier Science Publishers B.V.( North Holland ), 1990, 241-253.

[19] B. Šešelja, A. Tepavčević, Weak Congruences in Universal Algebra, Institute of mathematics Novi Sad, 2001. 
[20] A. Tepavčević, Diagonal relation as a continuous element in a weak congruence lattice, Proc. of the International Conference General Algebra and Ordered Sets, Olomouc, 1994, 156-163.

[21] A. Tepavčević, On special elements of bisemilattices, Novi Sad J. Math. Vol. 27, No. 1, 1997, 83-92.

[22] N.K. Thakare, S. Maeda, B.N. Waphare, Modular pairs, covering property and related results in posets, Journal of Indian Math. Soc. (to appear).

[23] N.K. Thakare, M.M. Pawar, B.N. Waphare, Modular pairs, Standard elements, neutral elements and related results in partially ordered sets (preprint).

[24] B. N. Waphare, V. Joshi, Characterizations of standard elements in posets (preprint).

Faculty of Technical Sciences, University of Kragujevac Svetog Save 65,32000 Cačak

Department of Mathematics and Informatics, University of Novi Sad Trg D. Obradovića 4, 21000 Novi Sad Serbia \& Montenegro 